\title{
Numerical Study on Phase-Fitted and Amplification-Fitted Diagonally Implicit Two Derivative Runge-Kutta Method for Periodic IVPs
}

(Kajian Berangka ke atas Suai-Fasa dan Suai-Pembesaran Kaedah Dua-terbitan Pepenjuru Tersirat Kaedah RungeKutta untuk MNA Berkala)

\author{
Norazak Senu, Nur Amirah Ahmad*, Zarina Bibi Ibrahim\& Mohamed Othman
}

\begin{abstract}
A fourth-order two stage Phase-fitted and Amplification-fitted Diagonally Implicit Two Derivative Runge-Kutta method (PFAFDITDRK) for the numerical integration of first-order Initial Value Problems (IVPs) which exhibits periodic solutions are constructed. The Phase-Fitted and Amplification-Fitted property are discussed thoroughly in this paper. The stability of the method proposed are also given herewith. Runge-Kutta (RK) methods of the similar property are chosen in the literature for the purpose of comparison by carrying out numerical experiments to justify the accuracy and the effectiveness of the derived method.
\end{abstract}

Keywords: Diagonally implicit methods; initial values problems; ordinary differential equations; phase-fitted and amplification-fitted; stability region; two derivative Runge-Kutta method

ABSTRAK

Kaedah Runge-Kutta Dua Terbitan Pepenjuru Tersirat Suai-Fasa dan Suai-Pembesaran (RKDTPTSFSP) tahap dua peringkat empat untuk penyelesaian pengamiran berangka Masalah Nilai Awal (MNA) peringkat pertama yang mengandungi penyelesaian berkala dibina. Sifat suai-fasa dan suai-pembesaran dibincangkan secara menyeluruh dalam kertas kajian ini. Kestabilan kaedah yang dicadangkan adalah seperti berikut. Kaedah Runge-Kutta (RK) dengan sifat yang sama dipilih di dalam kajian sorotan untuk tujuan perbandingan dengan menjalankan uji kaji berangka untuk memastikan kejituan dan keberkesanan kaedah yang diterbitkan.

Kata kunci: Kaedah pepenjuru tersirat; kaedah Runge-Kutta dua terbitan; masalah nilai awal; persamaan pembezaan biasa; rantau kestabilan; suai-fasa dan suai-pembesaran

\section{INTRODUCTION}

The Ordinary Differential Equations (ODEs) of first-order for the numerical solution of the IVPs are considered

$q^{\prime}=f(t, q)$, given the initial condition, $q(t)=q_{0},(1)$

where their solutions show periodically or oscillatory behavior in which the eigenvalue is in complex form. This type of problems appears throughout certain fields of applied sciences, for instance, mechanics, electronics, circuit simulation, orbital mechanics, astrophysics, and molecular dynamics. In general, periodically or oscillatory behavior problems are mostly known with second or higher order. It is therefore essential to perform order reduction to solve the ODEs (1) by reducing them to firstorder problems.

Anastassi and Simos (2012), Chen et al. (2012), and Kosti et al. (2012a) efficiently solved the Schrödinger equation and related periodically problems by designing a new explicit phase-fitted and amplification-fitted for the optimization of the method. 
RK methods for solving oscillatory problems using several techniques, for instance, phase-fitted and amplification-fitted, trigonometrically-fitted and exponentially-fitted techniques have been developed and expanded by several famous authors such as Simos (1998) in his written paper. Simos (1998) designed a RungeKutta method with exponentially-fitted properties for the numerical integration of IVPs of order five. Konguetsof and Simos (2003) introduced explicit symmetric multistep method which is exponentially-fitted and trigonometrically-fitted of eighth-order.

Recently, Adel et al. (2016) and Fawzi et al. (2015) derived two fourth-order modified RK and classical RK method with phase-fitted and amplification-fitted property, respectively. Meanwhile, Demba et al. (2016a, 2016b) suggested Runge-Kutta-Nyström (RKN) methods with trigonometrically-fitted property to solve secondorder IVPs with periodic solutions in nature derived on Simos' RKN method. Two Derivative Runge-Kutta (TDRK) methods which are explicit in nature given by Chan and Tsai (2010) in which they include the second derivative in its general formula making it special. Just one evaluation of function $f$ is involved along with a several number of function $g$ to be evaluated at every step. With this finding, they managed to derive methods up to order seven with five stages as well as some embedded pairs.

The numerical integration of radial Schrödinger equation and periodic problems are constructed by Zhang et al. (2013) using a TDRK method with trigonometrically-fitted of order five. Other than that, Fang et al. (2013) and Chen et al. (2015) constructed two TDRK methods of order four and three practical TDRK methods with exponentially-fitted, respectively. The newly derived methods are compared with some widely-known optimized codes as well as conventional RK methods with exponentially-fitted property mentioned in the literature.

In this recent year, there are no findings of research associated with phase-fitting and amplification-fitting in DITDRK methods. The benefits or drawbacks of applying phase-fitted and amplification-fitted property to DITDRK methods have not yet discussed thoroughly by researchers especially mathematicians. A two stage fourth-order DITDRK method with phase-fitted and amplification-fitted property is therefore derived in this paper. A summary of the TDRK method is discussed in Section 2. The next section considered the conditions for the phase-fitted and amplification-fitted property. The construction of the phase-fitted and amplification-fitted
DITDRK method is defined in Section 4. A description on the stability property is discussed briefly in Section 5 . The numerical results, discussion, and conclusion are presented briefly in Sections 6, 7, and 8, respectively.

\section{TWO DERIVATIVE RUNGE-KUTTA METHODS}

The scalar ODEs (1) is considered with $g: \Re^{N} \rightarrow \Re^{N}$. It is assumed, in this case, the second derivative is known where

$$
q^{\prime \prime}=g(q):=f^{\prime}(q) f(q), g: \mathfrak{R}^{N} \rightarrow \mathfrak{R}^{N} .
$$

The numerical integration of IVPs (1) for a TDRK method is given by

$$
\begin{aligned}
& q_{n+1}=q_{n}+\Delta t \sum_{i=1}^{s} b_{i} f\left(q_{i}\right)+\Delta t^{2} \sum_{i=1}^{s} \hat{b}_{i} g\left(Q_{i}\right), \\
& Q_{i}=q_{n}+\Delta t \sum_{j=1}^{s} a_{i j} f\left(q_{j}\right)+\Delta t^{2} \sum_{j=1}^{s} \hat{a}_{i j} g\left(Q_{j}\right),
\end{aligned}
$$

The lowest number of function evaluations for diagonally implicit methods can be established by considering the methods in the following manner

where $i=1, \ldots, \mathrm{s}$.

$$
\begin{aligned}
& q_{n+1}=q_{n}+\Delta t f\left(t_{n}, q_{n}\right)+\Delta t^{2} \sum_{i=1}^{s} \hat{b}_{i} g\left(t_{n}+\Delta t c_{i}, Q_{i}\right),(5) \\
& Q_{i}=q_{n}+\Delta t c_{i} f\left(t_{n}, q_{n}\right)+\Delta t^{2} \sum_{j=1}^{i} \hat{a}_{i j} g\left(t_{n}+\Delta t c_{j}, Q_{j}\right),(6)
\end{aligned}
$$

where $i=1, \ldots$, s.

Assume that all of the following DITDRK parameters $a_{i j}$, $\hat{a}_{i j}, b_{i i^{\prime}} \hat{b}_{i}$ and $c_{i}$ are real and $s$ is method's stages number. We introduced the -dimensional vectors $\mathrm{b}=\left[b_{1}, b_{2}, \ldots, b_{s}\right]^{T}, \hat{b}$ $=\left[\hat{b}_{1}, \hat{b}, \ldots, \hat{b}_{s}\right]^{T}, c=\left[c_{1}, c_{2}, \ldots, c_{s}\right]^{T}$ and $s \times s$ matrices A $=\left[a_{i j}\right]$ and $\hat{A}=\left[\hat{a}_{i j}\right]$ where $1 \leq i, j \leq s$. We use the following simplifying assumption,

$$
\sum_{j=1}^{s} \hat{a}_{i j}=\frac{1}{2} c_{i}{ }^{2},
$$

Table 1 shows the order conditions for unique DITDRK methods given in Chan and Tsai (2010). 


\begin{tabular}{|c|c|c|c|c|c|}
\hline Order & Conditions & & & & \\
\hline 1 & $b^{T} e=1$ & & & & \\
\hline 2 & $\hat{b}^{T} e=\frac{1}{2}$ & & & & \\
\hline 3 & $\hat{b}^{T} c=\frac{1}{6}$ & & & & \\
\hline 4 & $\hat{b}^{T} c^{2}=\frac{1}{12}$ & & & & \\
\hline 5 & $\hat{b}^{T} c^{3}=\frac{1}{20}$ & $\hat{b}^{T} \hat{A} c=\frac{1}{120}$ & & & \\
\hline 6 & $\hat{b}^{T} c^{4}=\frac{1}{30}$ & $\hat{b}^{T} c \hat{A} c=\frac{1}{180}$ & $\hat{b}^{T} \hat{A} c^{2}=\frac{1}{360}$ & & \\
\hline 7 & $\hat{b}^{T} c^{5}=\frac{1}{42}$ & $\hat{b}^{T} c^{2} \hat{A} c=\frac{1}{252}$ & $\hat{b}^{T} c \hat{A} c^{2}=\frac{1}{504}$ & $\hat{b}^{T} \hat{A} c^{3}=\frac{1}{840}$ & $\hat{b}^{T} \hat{A}^{2} c=\frac{1}{5040}$ \\
\hline
\end{tabular}

The method described herewith is identified as a unique DITDRK method. The remarkable aspect of this method is it requires just one evaluation of function $f$ and a few evaluations of function $g$ per step compared to a number of evaluations of function $f$ per step in the conventional RK methods. The following Butcher tableau illustrates the significant difference between the DITDRK method and the unique DITDRK method.

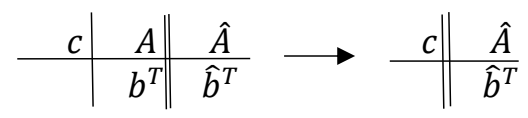

PHASE-FITTED AND AMPLIFICATION-FITTED PROPERTY

The following linear scalar equation is considered,

$$
q^{\prime}=i \lambda q
$$

The exact solution with initial value $q\left(t_{0}\right)=q_{0}$ of this equation satisfies

$$
q\left(t_{0}+\Delta t\right)=H_{0}(z) q_{0}
$$

where $H_{0}(z)=\exp (z), z=i v$. A phase advance $v=$ $\lambda \Delta \mathrm{t}$ is experienced by the exact solution whereby the amplification appears to remain stable and secure after a cycle of time $\Delta \mathrm{t}$.

The DITDRK method is adapted to the test equation (8) to yield

$$
q_{1}=H(z) q_{0}
$$

where

$$
H(z)=\left(1+v^{2} \hat{b}\left(I-v^{2} \hat{A}\right)^{-1} e\right)+i\left(v+v^{3} \hat{b}\left(I-v^{2} \hat{A}\right)^{-1} c\right),(11)
$$

where $e=[1, \ldots, 1]^{T}$.

The stability function of the DITDRK method is presented by $H(z)$ which in term of complex number. The function is split in terms of the real (denoted as $U(v)$ ) and imaginary (denoted as $U(v)$ ) part of $H(z)$, Further, we have the argument of $H(z)$ or simply $\arg H(z)=\tan ^{-1}\left(\frac{V(v)}{U(v)}\right)$ and the magnitude of $H(z)$ or $|H(z)|=\sqrt{U^{2}(v)+V^{2}(v)}$ for small $\Delta \mathrm{t}$. According to the analysis above, the following definition arises. 
Definition 1 (van der Houwen \& Sommeijer (1987)) The quantities

$$
\tilde{P}(v)=v-\arg H(z), \widetilde{D}(v)=1-|H(z)|,
$$

are defined as the phase lag (or dispersion) and the error of amplification factor (or dissipation) of the method, respectively. If

$$
\tilde{P}(v)=c_{\phi} v^{\alpha+1}+\mathcal{O}\left(v^{\alpha+3}\right), \widetilde{D}(v)=c_{d} v^{\beta+1}+\mathcal{O}\left(v^{\beta+3}\right)
$$

then, the method is defined as dispersive of order $\alpha$ and dissipative of order $\beta$, respectively.

If

$$
\widetilde{P}(v)=0, \widetilde{D}(v)=0,
$$

the method is defined as phase-fitted (or zero-dispersive) and amplification-fitted (or zero dissipative), respectively. Theorem 2 (Chen et al. 2012)

The method is justified as phase-fitted and amplificationfitted if and only if

$$
U(v)=\cos (v), V(v)=\sin (v) .
$$

The local truncation error (LTE), LTE $=q\left(t_{0}+\Delta t\right)=\mathcal{O}(\Delta t$ $\left.\zeta^{+1}\right)$, for any $(\zeta+1$-th differentiable function $g(q)$, when equations (5) and (6) are applied to the first-order ODEs (1). Thence, the method is said to have a (algebraic) order $\zeta$. Define

$$
E C_{\zeta+1}(v)=\left(\sum_{i=1}^{j}\left(\tau_{j}^{(\zeta+1)}\right)^{2}\right)^{\frac{1}{2}}
$$

where $\tau_{j}^{(\zeta+1)}$ is the error coefficient of the method. The nonnegative number

$$
E C_{\zeta+1}=\lim _{v \rightarrow 0} E C_{\zeta+1}(v)
$$

is known as the method's error constant.

\section{DERIVATION OF THE NEW PHASE-FITTED AND AMPLIFICATION-FITTED METHOD}

If and only if Theorem 2 is satisfied, then only a DITDRK method appeared to be phase-fitted and amplification-fitted. Thus, the proposed method is derived by combining the DITDRK method with the phase-fitted and amplificationfitted property proposed in this section.

First, a fourth-order two stages DITDRK method will be derived. Referring to the order conditions in Table 1 up to fourth-order, we have

$$
\begin{gathered}
\hat{b}_{1}+\hat{b}_{2}-\frac{1}{2}=0, \\
\hat{b}_{1} c_{1}+\hat{b}_{2} c_{2}-\frac{1}{6}=0, \\
\hat{b}_{1} c_{1}{ }^{2}+\hat{b}_{2} c_{2}{ }^{2}-\frac{1}{12}=0 .
\end{gathered}
$$

Solving equation (18)-(20) we obtain $\widehat{b}_{1} \widehat{b}_{2}$ and $c_{1}$ in term of $c_{2}$

$$
\begin{gathered}
\hat{b}_{1}=\frac{1}{\left(36 c_{1}{ }^{2}-24 c_{1}+6\right)^{\prime}}, \\
\hat{b}_{2}=\frac{1}{3}\left(\frac{9 c_{1}{ }^{2}-6 c_{1}+1}{6 c_{1}{ }^{2}-4 c_{1}+1}\right), \\
c_{2}=\frac{1}{2}\left(\frac{2 c_{1}-1}{3 c_{1}-1}\right) .
\end{gathered}
$$

Our main focus is to choose $c_{1}$ in such a way that a very small value of the principal local truncation error coefficient, $\left\|\tau^{(5)}\right\|_{2}$ might be achieved. There will be a significant global error difference with an inaccurate choice of $c_{1}$. The graph of $\left\|\tau^{(5)}\right\|_{2}$ against $c_{1}$ is plotted in Figure 1 where a small value of $c_{1}$ is chosen within the range of $[0.0,1.0]$. Therefore, the value of $c_{1}$ is between $[0.1,0.3]$ with the help of Maple software where we use the minimisation command for non-linear functions. For simplicity, we have chosen $c_{1}=\frac{1}{5}$ for an ideal optimized pair by running empirical experiment.

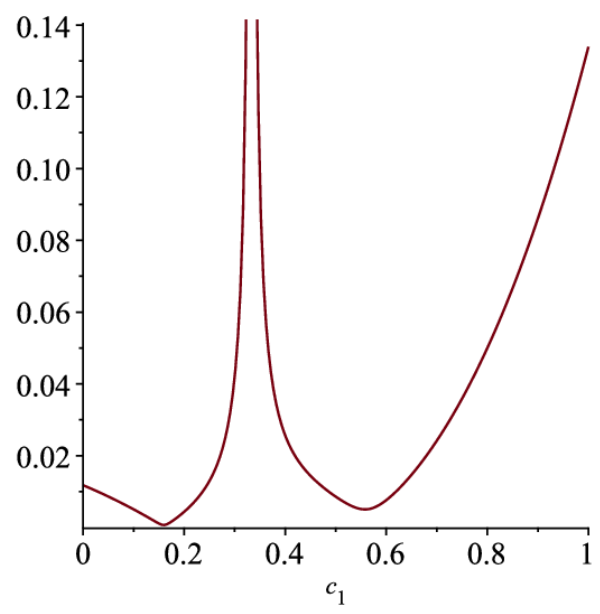

FIGURE 1. The graph of $\left\|\tau^{(5)}\right\|_{2}$ against $c_{1}$ 
The following Butcher tableau represents the coefficients

TABLE 2. Butcher Tableau for DITDRK $(2,4)$ Method

\begin{tabular}{c||cc}
$\frac{1}{5}$ & $\frac{1}{50}$ & \\
$\frac{3}{4}$ & $\frac{209}{800}$ & $\frac{1}{50}$ \\
\hline & $\frac{25}{66}$ & $\frac{4}{33}$
\end{tabular}

The stability function (11) for two stages fourth-order DITDRK method is considered. Therefore, by choosing $\hat{a}_{21}$ and $c_{1}$ as free parameters, we have

$I=\left[\begin{array}{ll}1 & 0 \\ 0 & 1\end{array}\right], e=\left[\begin{array}{l}1 \\ 1\end{array}\right], \hat{b}=\left[\begin{array}{c}\frac{25}{66} \\ \frac{4}{33}\end{array}\right], c=\left[\begin{array}{c}c_{1} \\ \frac{3}{4}\end{array}\right], \hat{A}=\left[\begin{array}{cc}\frac{1}{50} & \\ \hat{a}_{21} & \frac{1}{50}\end{array}\right]$

We substituted the matrices (24) into $H(z)$ given by equation (11) and splitted the complex number of $H(z)$ into real and imaginary as mentioned. The free parameters, $\hat{a}_{21}$ and $c_{1}$ are taken as the ideal combination for the optimized value of the maximum global error. By implementing Theorem 2, (15) is solved to get the coefficients of $\hat{b}_{1}$ and $c_{4}$ and this resulting in

$$
\cos (v)=\frac{1}{33} \frac{10000 v^{4} \hat{a}_{21}-792 v^{4}-37950 v^{2}+82500}{\left(v^{2}+50\right)^{2}}
$$

$\sin (v)$

$$
=\frac{1}{33} \frac{v\left(10000 v^{4} \hat{a}_{21} c_{1}-625 v^{4} c_{1}-117 v^{4}-31250 v^{2} c_{1}-4200 v^{2}+82500\right)}{\left(v^{2}+50\right)^{2}} .
$$

By solving (25) and (26) we will obtain the following

$\hat{a}_{21}$

$=\frac{(33 \cos (v)+792) v^{4}+(3300 \cos (v)+37950) v^{2}+82500 \cos (v)-82500}{10000 v^{4}}$,

$$
c_{1}=\frac{33 \sin (v) v^{2}+117 v^{3}+1650 \sin (v)-1650 v}{33 \cos (v) v^{3}+167 v^{3}+1650 \cos (v) v-1650 v} .
$$

The following Taylor expansions as $v \rightarrow 0$ are obtained as follows

$$
\begin{gathered}
\hat{a}_{21}=\frac{209}{800}+\frac{77 v^{2}}{120000}-\frac{781 v^{4}}{6720000}+\frac{803 v^{6}}{604800000}+\frac{59 v^{8}}{7257600000}-\frac{2081 v^{10}}{6604416000000} \\
+\cdots, \\
c_{1}=\frac{1}{5}+\frac{11 v^{2}}{3125}+\frac{3476 v^{4}}{41015625}+\frac{13111549 v^{6}}{3691406250000}+\frac{1679796241 v^{8}}{9228515625000000} \\
+\frac{66016889468987 v^{10}}{6298461914062500000000}+\cdots
\end{gathered}
$$

The following expansions shall be obtained by direct calculation:

$\hat{b}^{T} e=\frac{1}{2}$

$\hat{b}^{T} c=-\frac{5}{66}+\frac{825 \sin (v) v^{2}+2925 v^{3}+41250 \sin (v)-41250 v}{2178 \cos (v) v^{3}+11022 v^{3}+108900 \cos (v) v-108900 v}$

$$
=\frac{1}{6}+\mathcal{O}(v)
$$

$\hat{b}^{T} c^{2}=-\frac{1}{66}+\frac{25}{66}\left(\frac{M}{N}\right)^{2}=\frac{1}{12}+\mathcal{O}(v)$.

Subsequently, all of the order conditions till order four are satisfied by the coefficients shown in Table 2. But the condition for order five was not satisfied. For instance,

$$
\hat{b}^{T} c^{3}=0 \neq \frac{1}{20}+\mathcal{O}(v)
$$

Therefore, it is a method of order four. The coefficients of error of the DITDRK $(2,4)$ for order five are given by

$$
\tau_{1}^{(5)}=\frac{1}{240}, \quad \tau_{2}^{(5)}=\frac{1}{750} .
$$


Therefore, for DITDRK $(2,4)$, we obtain the following

$$
E C_{5}=\frac{1}{6000} \sqrt{689}
$$

As we have proven that this newly derived method is fourth-order, it is therefore known as PFAFDITDRK $(2,4)$. The error coefficients of PFAFDITDRK $(2,4)$ are given by

$$
\begin{aligned}
\tau_{1}^{(5)} & =\frac{1}{880}+\frac{25}{66}\left(\frac{M}{N}\right)^{3} \\
\tau_{2}^{(5)} & =\left(\frac{1}{132}+\frac{1}{82500 v^{4}}\right)\left(\frac{M P}{N}\right)-\frac{43}{6600}
\end{aligned}
$$

For PFAFDITDRK $(2,4)$, we have

$$
\begin{aligned}
& E C_{5}(v)=\left(\frac { 1 } { 1 0 8 9 0 0 0 0 0 0 0 0 N ^ { 6 } } \left(15625000000 M^{6}+93750000(M N)^{3}\right.\right. \\
&\left.+6250000\left(M N^{2}\right)^{2}-10750000 M N^{5}+4763125 N^{6}\right) \\
&+\frac{1}{108900000000 N^{6} v^{4}}\left(20000\left(M N^{2}\right)^{2} P-17200 M N^{5} P\right) \\
&\left.+\frac{1}{6806250000 v^{8}}\left(\frac{M P}{N}\right)^{2}\right)^{\frac{1}{2}}
\end{aligned}
$$

where $M=33(v) v^{2}+117 v^{3}+1650 \sin (v)-1650 \mathrm{v}, N=$ $33 \cos (v) v^{3}+167 v^{3}+1650 \cos (v) v-1650 v, P=33 \cos$ (v) $v^{4}+792 v^{4}+3300 \cos (v) v^{2}+37950 v^{2}+82500 \cos$ (v) -82500 .

PFAFDITDRK $(2,4)$ will reduce to its actual method, $\operatorname{DITDRK}(2,4)$ as $v \rightarrow 0$. Apart from that, $\operatorname{PFAFDITDRK}(2,4)$ will have the identical error constant as $\operatorname{DITDRK}(2,4)$ as $v \rightarrow 0$.

STABILITY AND CONVERGENCE OF THE NEW METHOD The linear stability of the method being developed is analysed in this section. Applying equation (8) to the DITDRK method produces the difference equation

$$
q_{n+1}=H(z) q_{n}, z=i v, i^{2}=-1
$$

where $H(z)$ is given as (11).

Definition 3 A DITDRK method is said to be absolutely stable if $|H(z)|<1$ for all $z \in(-v, 0)$. The stability polynomial of the PFAFDITDRK $(2,4)$ method is shown as follows.

$$
\begin{gathered}
H(v)=\frac{1}{49883818359375000000000\left(v^{2}-50\right)^{2}}\left(56936760886197823 v^{15}\right. \\
-4763031005859375 v^{14}+1022777450921523750 v^{13}+ \\
122886657714843750 v^{12}+17921737015284375000 v^{11}+ \\
20070098876953125000 v^{10}+105179969425781250000 v^{9} \\
-1756820983886718750000 v^{8}+ \\
9699631347656250000000 v^{6}+590291850585937500000000 v^{5}+ \\
57366391113281250000000000 v^{2}+124709545898437500000000000 v+ \\
124709545898437500000000000+\cdots) .
\end{gathered}
$$

We plot and compare the region of stability of the PFAFDITDRK $(2,4)$ method up to $v^{i}, i=6,8,14$ and its actual method as in Figure 2.

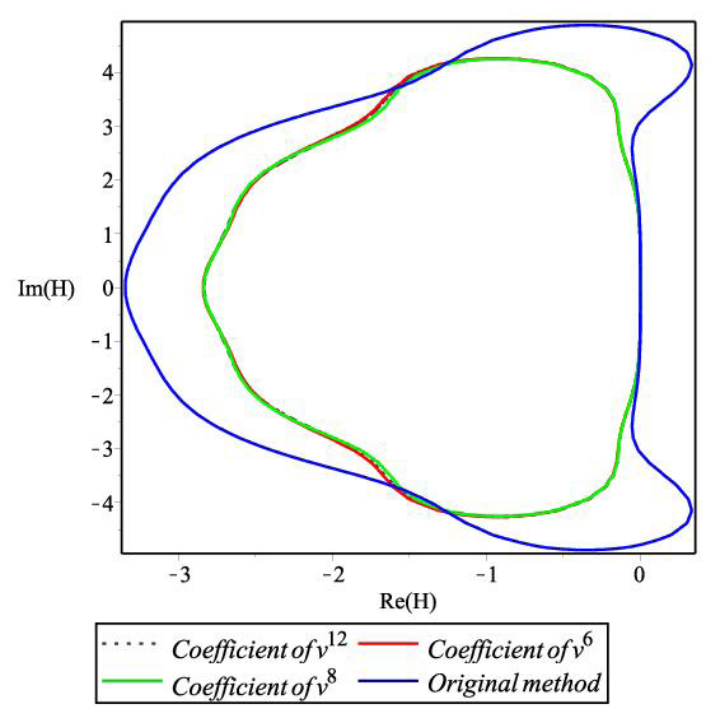

FIGURE 2. Stability region of PFAFDITDRK $(2,4)$ method for different order 
The stability interval with the coefficients $v^{6}, v^{8}$ and $v^{12}$ of this method are $(-2.843,0.000),(-2.837,0.000)$ and $(-2.833,0.000)$, respectively. The stability regions in Figure 2 is observed and as the coefficients order tends to infinity, the stability interval becomes further away from the original method where it is given by $(-3.347,0.000)$.

Through the stability interval, we can literally consider the largest value of $\Delta t$ the method could take to ensure it will remain stable. $v=\lambda \Delta t$ is mentioned earlier and the test problems represents the value of $\lambda$. Therefore, the value of $\Delta t$ is obtained by dividing $v$ with $\lambda$. The stability test as following would illustrate on how the regions of stability are used for practical purposes. We have given that $\varphi(t)$ is a smooth function. Letting $\lambda=-1, \varphi(t)$ $=\sin (t)$ and $q(t)=\varphi(t)$ is the exact solution.

Stability of the method can be achieved once the maximum global error is small enough and therefore converging to its exact solution. Instead of that, a larger maximum global error indicates that the method is unstable, meaning that they are actually diverging from its exact solution. The stability test will be conducted to demonstrate the connection between $\Delta t, \lambda$ and $|H(z)|$. When $\Delta t=4.15$, the stability is achieved whereby this is the largest value of $\Delta t$ can be used to ensure the method remain stable in this particular test for stability. Table 3 represents the global error for a variety of $\Delta t$ values.

$q^{\prime}=\lambda(q-\varphi)+\varphi^{\prime}, q(0)=\varphi(0), \operatorname{Re}(\lambda)<0, t \in[0,2000]$,

TABLE 3. Stability test for PFAFDITDRK $(2,4)$ using coefficient of $v^{8}$ with $\lambda=-1$ for variable $\Delta t$

\begin{tabular}{ccc}
\hline$\Delta \mathbf{t}$ & $|\mathbf{H}(\mathbf{z})|$ & Global Error \\
\hline 3.20 & 2.399948118 & $1.729241 \times 10^{236}$ \\
3.00 & 1.496894148 & $5.112085 \times 10^{114}$ \\
2.83 & 0.9808945284 & $4.720623 \times 10^{0}$ \\
1.00 & 0.3650531765 & $1.836955 \times 10^{(-3)}$ \\
0.15 & 0.8607077753 & $5.005154 \times 10^{(-7)}$ \\
0.01 & 0.9900498337 & $9.473644 \times 10^{(-12)}$ \\
\hline
\end{tabular}

Definition 4 (Henrici, 1962)

The numerical method with order $p$ is zero stable if numerical solutions remain bounded in the limit $\Delta t \rightarrow$ 0 , with the modulus of roots for the first characteristic polynomial are less than or equal to zero.

In studying the zero stability of the DITDRK method, the characteristic polynomial of method (5)-(6) is:

$$
p(\xi)=(\xi-1)
$$

Hence, the method is zero stable since the roots, $\xi=1$ are less than or equal to one.
Definition 5 (Suli \& Mayers 2003)

The method is consistent with the order at least $p$ if and only if local truncational error, $T_{p+1}=\mathcal{O}\left(\Delta t^{p+1}\right)$ as $\Delta \mathrm{t} \rightarrow 0$. Consider DITDRK methods in the class as follow:

$$
\sum_{j=0}^{s} \delta_{j} q_{n+j}=\Delta t \gamma_{j} f_{n+j}+\Delta t^{2} \phi g\left(q_{n+k}, q_{n+k-1}, \ldots, q_{n}, t_{n} ; \Delta t^{2}\right) \text {. }
$$

On putting $s=1$, then

$$
\begin{gathered}
\delta_{1}=1, \delta_{0}=-1, \gamma_{0}=1, \quad \phi g\left(q_{n}, t_{n} ; \Delta t^{2}\right)=\sum_{i=1}^{s} \hat{b}_{i} Q_{i}, \\
Q_{i}=q_{n}+\Delta t c_{i} f\left(t_{n}, q_{n}\right)+\Delta t^{2} \sum_{j=1}^{i} \hat{a}_{i j} g\left(t_{n}+\Delta t c_{j}, Q_{j}\right),
\end{gathered}
$$


where $i=1, \ldots, s$.

The condition for (39) to be consistent are

$$
\begin{gathered}
\sum_{j=0}^{s} \delta_{j}=0, \sum_{j=0}^{s}\left(j \delta_{j}-\gamma_{j}\right)=0, \\
\frac{\phi g\left(q\left(t_{n}\right), q\left(t_{n}\right), \ldots, q\left(t_{n}\right), t_{n} ; 0\right)}{\sum_{j=0}^{s} j \delta_{j},}=g\left(t_{n}, q\left(t_{n}\right)\right) .
\end{gathered}
$$

Applying the conditions (40), the necessary and sufficient condition for DITDRK methods to acquire consistency is

$$
\phi g\left(q\left(t_{n}\right), t_{n} ; 0\right)=g\left(t_{n}, q\left(t_{n}\right)\right) \Leftrightarrow \sum_{i=0}^{s} \hat{b}_{i}=\frac{1}{2} \text {. }
$$

Here, local truncation error, $T_{n+1}$ at $t_{n+1}$ at is expressed as the residual when $q_{n+j}$ is replaced by $q\left(t_{n+j}\right)$ which is

$$
\begin{aligned}
T_{n+1}=q\left(t_{n+1}\right) & +\Delta t f\left(t_{n}, q\left(t_{n}\right)\right)-\left[q\left(t_{n}\right)+\Delta t f\left(t_{n+1}, q\left(t_{n+1}\right)\right)\right] \\
& -\Delta t^{2} \phi g\left(q\left(t_{n}\right), t_{n} ; \Delta t^{2}\right),
\end{aligned}
$$

where $\phi g$ is defined in (39). Assuming that $p$ is the largest integer whereby $T_{n+1}=\mathcal{O}\left(\Delta t^{p+1}\right)$, then the method has order $p$ (Lambert 1991). We denote by $\tilde{q}_{n+1}$ the value at $t_{n+1}$ generated by DITDRK method when the localising assumption, $q_{n}=q\left(t_{n}\right)$ is made. Since

$$
\tilde{q}_{n+1}=q_{n}+\Delta t f\left(t_{n}, q_{n}\right)+\Delta t^{2} \phi g\left(q_{n}, t_{n} ; \Delta t^{2}\right) .
$$

Then we have

$$
q\left(t_{n+1}\right)-\tilde{q}_{n+1}=T_{n+1}
$$

DITDRK method is consistent if they follow (41) such that

$$
\begin{array}{r}
q\left(t_{n+1}\right)+\Delta t f\left(t_{n}, q\left(t_{n}\right)\right)-\left[q\left(t_{n}\right)+\Delta t f\left(t_{n+1}, q\left(t_{n+1}\right)\right)\right] \\
=\frac{\Delta t^{2}}{2} f^{\prime}\left(t_{n}\right)-\frac{\Delta t^{2}}{2} g\left(t_{n}, q\left(t_{n}\right)\right)+\mathcal{O}\left(\Delta t^{3}\right)
\end{array}
$$

By reason of $f^{\prime}\left(t_{n}\right)=g\left(t_{n}, q\left(t_{n}\right)\right), T_{n+1}$ for DITDRK method is equal to $\mathcal{O}\left(\Delta t^{3}\right)$, it shows that DITDRK method is consistent if their order is at least 2, which is in line with our definitions of order for linear multistep methods. Since the order of DITDRK method is at least 2, and hence, this method is consistent.

Convergence is a property of numerical method related to truncation errors that ensures the numerical solution converges onto the exact solution and the global truncation error goes to zero at all step size indices in the limit $\Delta \mathrm{t} \rightarrow 0$ (Atkinson 2009). Maximum absolute global truncation error between the analytical solution and numerical solution the gets smaller as the step size becomes lesser.

Definition 6 (Lambert 1991)

The numerical method is convergent if acquiring the properties of zero stability and consistency.

Since DITDRK method is zero-stable and consistent, implies that DITDRK method is convergent.

\section{PROBLEMS TESTED AND NUMERICAL RESULTS}

The derived method PFAFDITDRK $(2,4)$ are compared in term of their numerical performances with some famous existing RK and TDRK methods by considering Problems 1-5 as follows. C Programming codes are used for solving differential equations where all the problems chosen are having oscillatory solutions.

Problem 1 (Harmonic Oscillator)

$$
\begin{aligned}
& q_{1}^{\prime}(t)=q_{2}(t), \quad q_{1}(0)=q_{0_{1}}, \quad t \in\left[0, t_{\text {end }}\right] \\
& q_{2}^{\prime}(t)=-\omega^{2} q_{1}(t), \quad q_{2}(0)=q_{0_{2}} .
\end{aligned}
$$

Exact solution is

$$
\begin{aligned}
& q_{1}(t)=\bar{c}_{1} \sin (\omega t)+\bar{c}_{2} \cos (\omega t) \\
& q_{2}(t)=\bar{c}_{3} \omega \cos (\omega t)-\bar{c}_{4} \omega \sin (\omega t)
\end{aligned}
$$

Total energy as given in Pokorny (2009)

$$
E\left(q_{1}, q_{2}\right)=\frac{q_{1}^{2}}{2}+\frac{q_{2}^{2}}{2}=\frac{\Psi^{2}}{2}
$$

where $\Psi$ depends on the initial conditions.

Problem 2 (Inhomogeneous problem (Van de Vyver 2007))

$$
\begin{aligned}
& q_{1}^{\prime}=q_{2}, \quad q_{1}(0)=1, \quad t \in[0,1000], \\
& q_{2}{ }^{\prime}=-100 q_{1}+99 \sin (t), \quad q_{2}(0)=11 .
\end{aligned}
$$

Exact solution is

$$
\begin{gathered}
q_{1}(t)=\cos (10 t)+\sin (10 t)+\sin (t), \\
q_{2}(t)=-10 \sin (10 t)+10 \cos (10 t)+\cos (t) .
\end{gathered}
$$

Problem 3 (An almost Periodic Orbit problem (Stiefel \& Bettis 1969)) 


$$
\begin{aligned}
& q_{1}{ }^{\prime}=q_{2}, \quad q_{1}(0)=1, \quad t \in[0,1000], \\
& q_{2}{ }^{\prime}=-q_{1}+0.001 \cos (t), \quad q_{2}(0)=0, \\
& q_{3}{ }^{\prime}=q_{4}, \quad q_{3}(0)=0, \\
& q_{4}{ }^{\prime}=-q_{3}+0.001 \sin (t), \quad q_{4}(0)=0.9995 .
\end{aligned}
$$

Exact solution is

$q_{1}(\mathrm{t})=\cos (t)+0.0005 t \sin (t), q_{2}(t)=-\sin (t)+0.0005 t$ $\cos (t)+0.0005 t \sin (t)$

$\left.q_{3}(\mathrm{t})=\sin (t)-0.0005 \mathrm{t} \cos (\mathrm{t}), q_{4}(t)=\cos t\right)+0.0005 t$ $\sin (t)-0.0005 \cos (t)$.

Problem 4 (Duffing problem (Kosti et al. 2012b)

$q_{1}{ }^{\prime}=q_{2}, q_{1}(0)=0.200426728067$,

$q_{2}{ }^{\prime}=-q_{1}-q_{1}{ }^{3}+0.002 \cos (1.01 t), q_{2}(0)=0, t \in[0,1000]$.

Exact solution is

$q_{1}(t)=0.200179477536 \cos (1.01 t)+2.46946143 \times 10^{-4}$ $\cos (3.03 \mathrm{t})+3.04014 \times 10^{-7} \cos (5.05 t)+3.74 \times 10^{-10} \cos$ $(7.07 t)$, $q_{2}(t)=-0.2021812723 \sin (1.01 t)-7.482468133 \times 10^{-4}$ $\sin (3.03 t)-1.53527070 \times 10^{-6} \sin (5.05 \mathrm{t})-2.64418 \times 10^{-9}$ $\sin (7.07 t)$.

Problem 5 (Prothero-Robinson problem Chan \& Tsai 2010) $q^{\prime}=\lambda(q-\varphi)+\varphi^{\prime}, q(0)=\varphi(0), \operatorname{Re}(\lambda)<0, t \in[0,1000]$,

where $\varphi(t)$ is a smooth function and taking $\lambda=-1, \varphi(t)$ $=\sin (t)$.

Exact solution is $q(t)=\varphi(t)$.

Figures 3-18 used the following abbreviations.

PFAFDITDRK $(2,4)$ : Fourth-order two stages phase-fitted and amplification-fitted DITDRK method proposed in this paper. TFDIRKK $(3,4)$ : Fourth-order three stages trigonometrically-fitted DIRK method developed in Kalogiratou (2013). PFAFDIRKA(3,4): Fourth-order three stages phase-fitted and amplification-fitted DIRK method given by Ahmad et al. (2016). EFDIRKE(3,4): Fourth-order three stages exponentially-fitted DIRK method given in Ehigie et al. (2018).

Figures 3-18 represents the behaviour of these numerical results in graphics form.

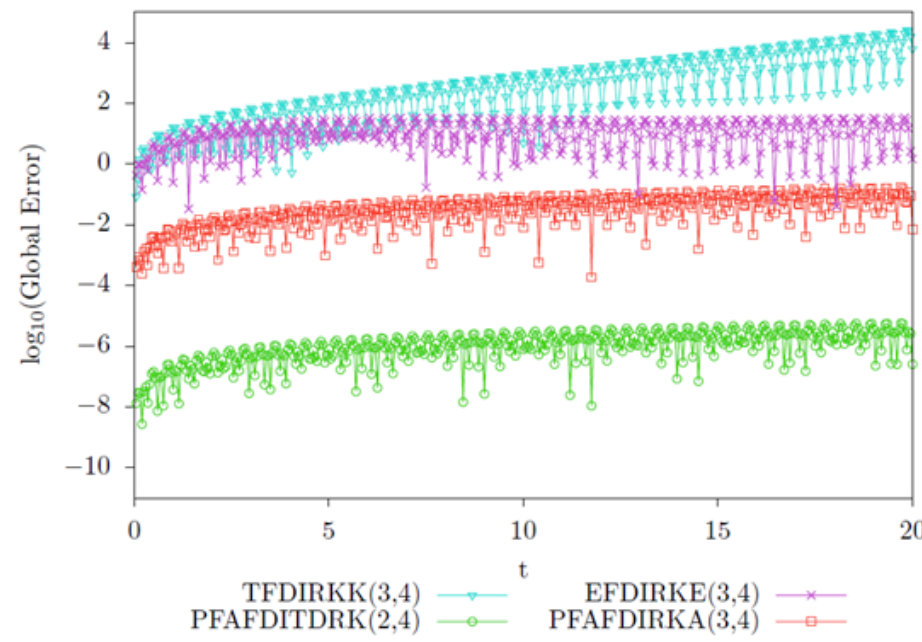

FIGURE 3. (Conservation of Energy). The logarithm error of energy (Global Error) when solving the harmonic oscillator (Problem 1) at each integration point for $\omega=8, q_{01}=1, q_{02}=-2$ and $\Delta \mathrm{t}=1 / 20$ 


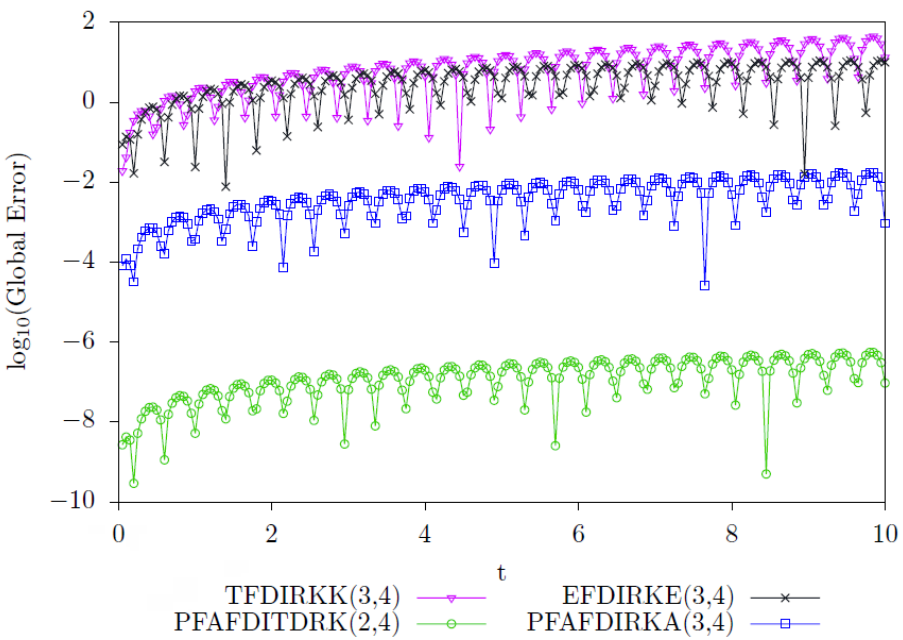

FIGURE 4. The error when solving the harmonic oscillator (Problem 1) at each integration point where $\omega=8, q_{01}=1, q_{02}=-2$ and $\Delta t=1 / 20$

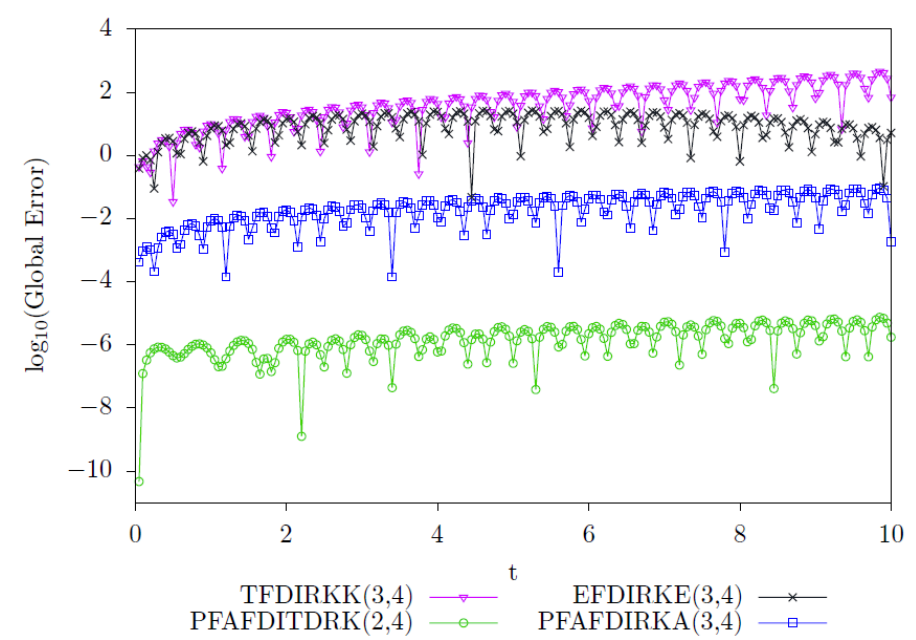

FIGURE 5. The global error when solving the inhomogeneous problem (Problem 2) at each integration point where $\Delta t=1 / 20$

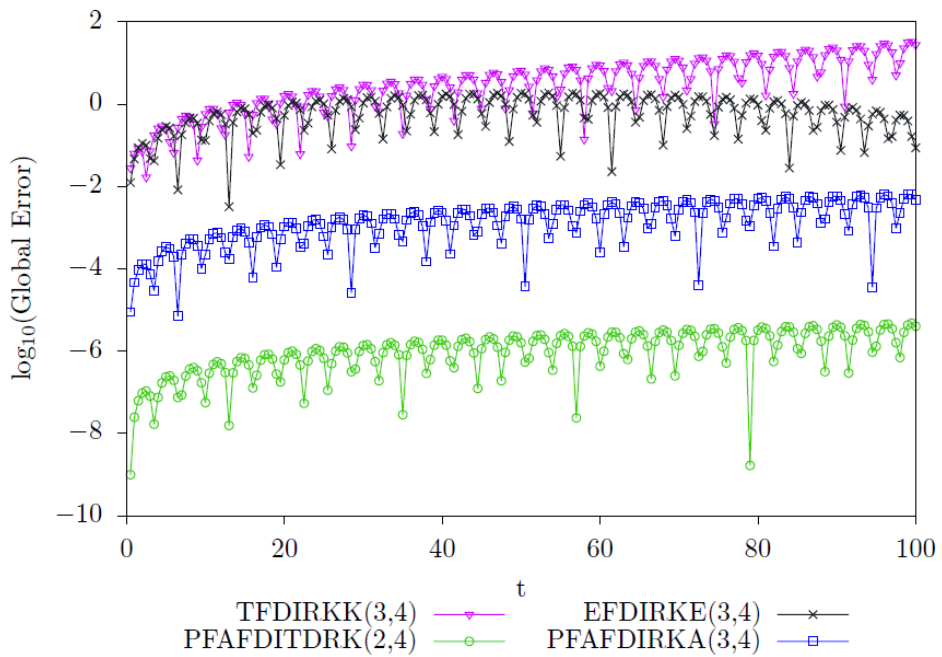

FIGURE 6. The global error when solving the almost periodic problem (Problem 3 ) at each integration point where $\Delta \mathrm{t}=1 / 2$ 


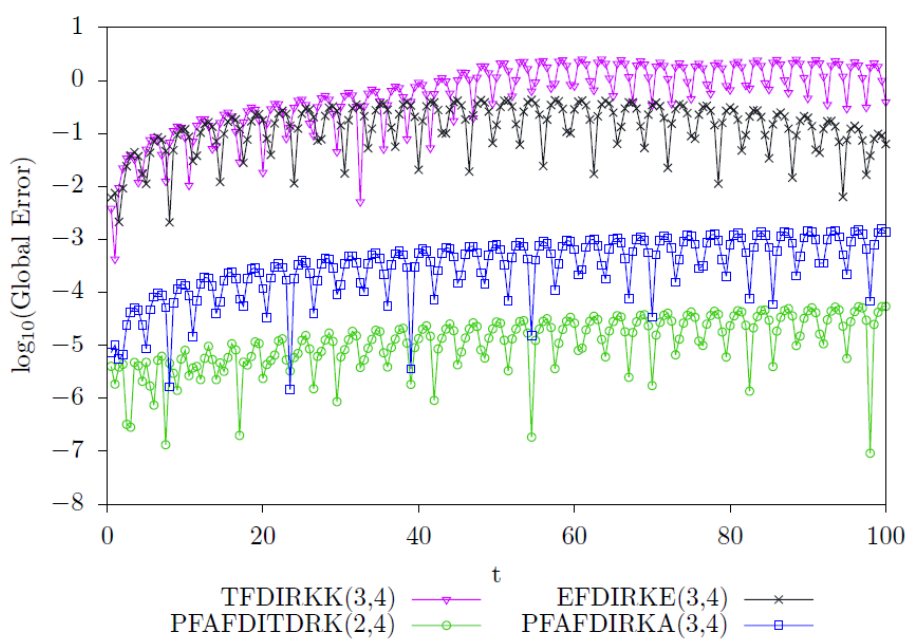

FIGURE 7. The global error when solving the Duffing problem

(Problem 4) at each integration point where $\Delta t=1 / 2$

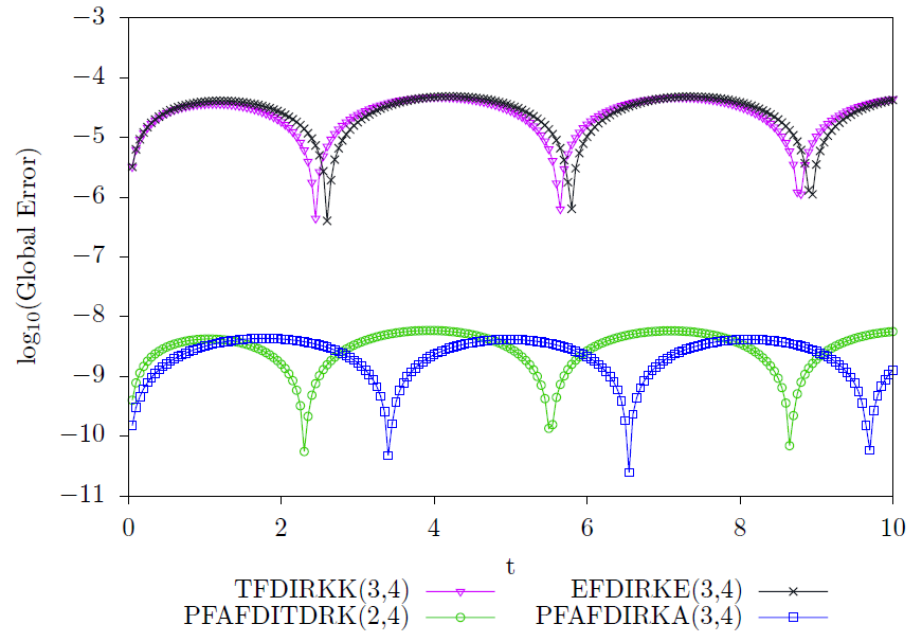

FIGURE 8. The global error when solving the Prothero-Robinson problem (Problem 5) at each integration point where $\Delta t=1 / 20$

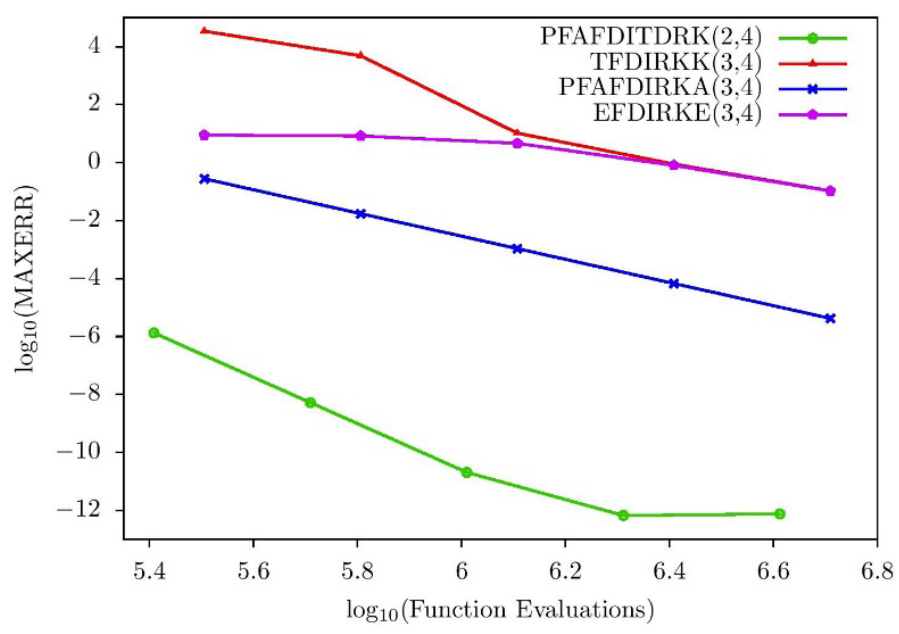

FIGURE 9. The curve for the harmonic oscillator (Problem 1) with $\lambda=8, \Delta \mathrm{t}=1.0 / 2^{i}, i=5, \ldots, 9$ with $\mathrm{t}_{\text {end }}=1000$ 


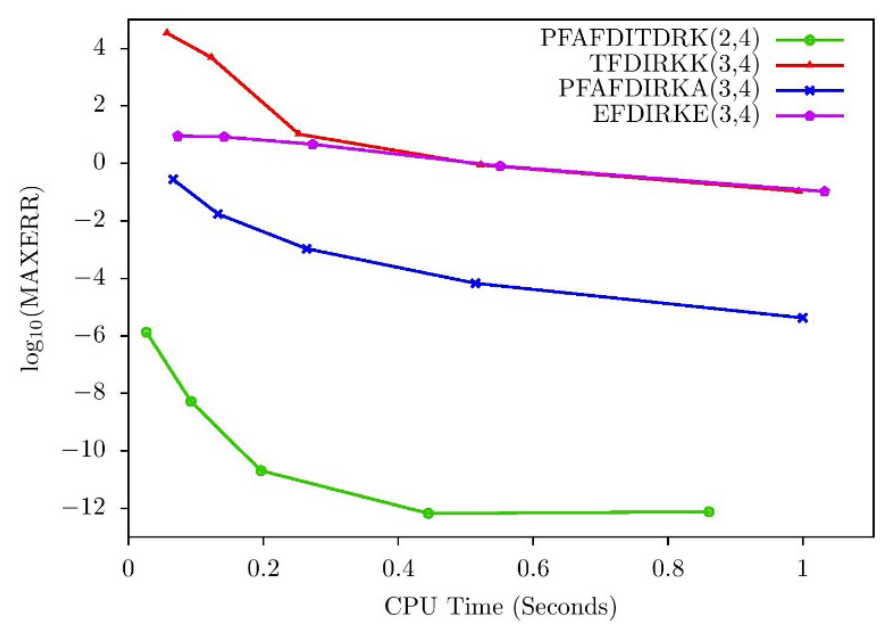

FIGURE 10. The curve for the harmonic oscillator (Problem 1) with $\lambda=8, \Delta \mathrm{t}=1.0 / 2^{i}, i=5, \ldots, 9$ with $\mathrm{t}_{\text {end }}=1000$

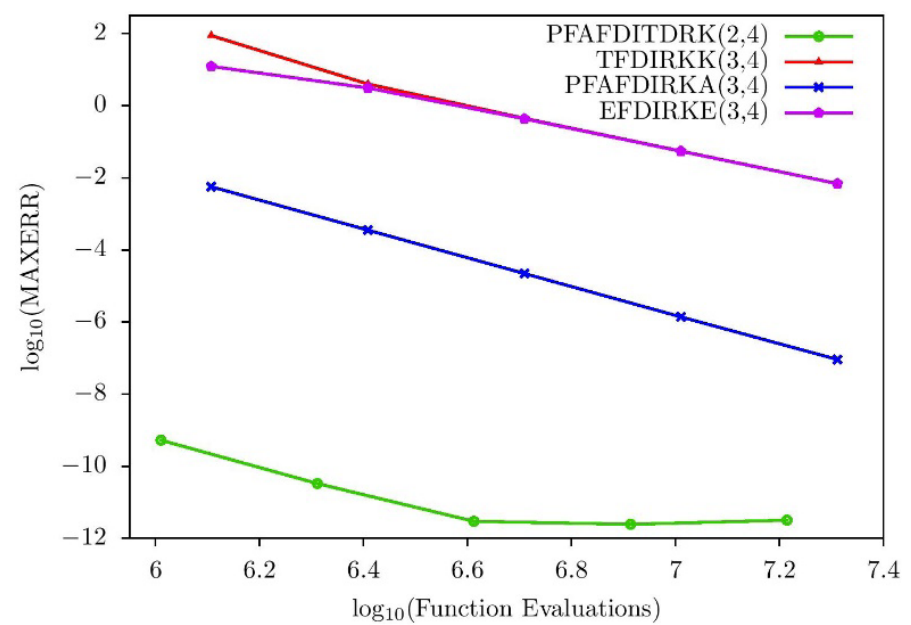

FIGURE 11. The curve for the inhomogeneous problem (Problem 2) with time step $\Delta \mathrm{t}=1.0 / 2^{i}, i=7, \ldots, 11$

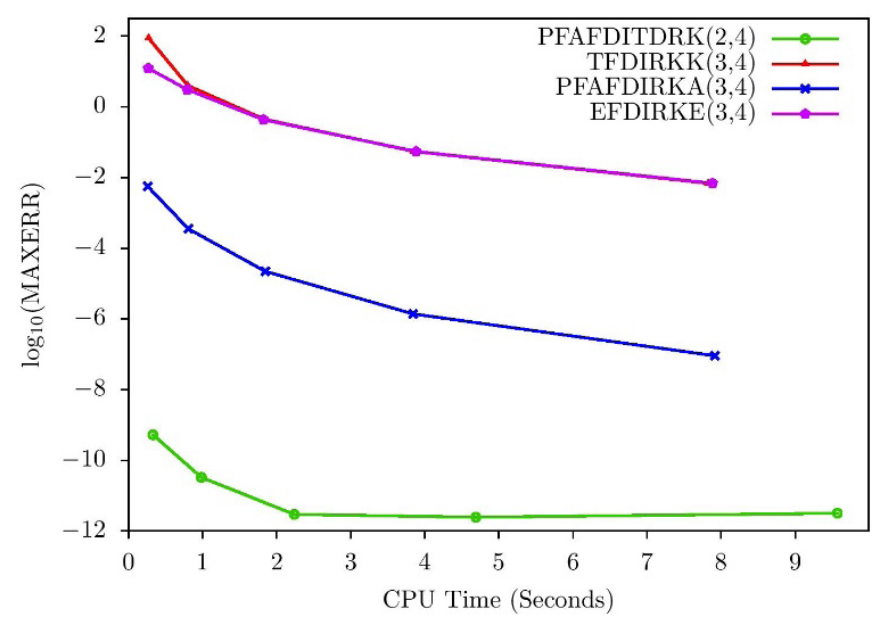

FIGURE 12.The curve for the inhomogeneous problem (Problem 2) with time step $\Delta \mathrm{t}=1.0 / 2^{i}, i=7, \ldots, 11$ 


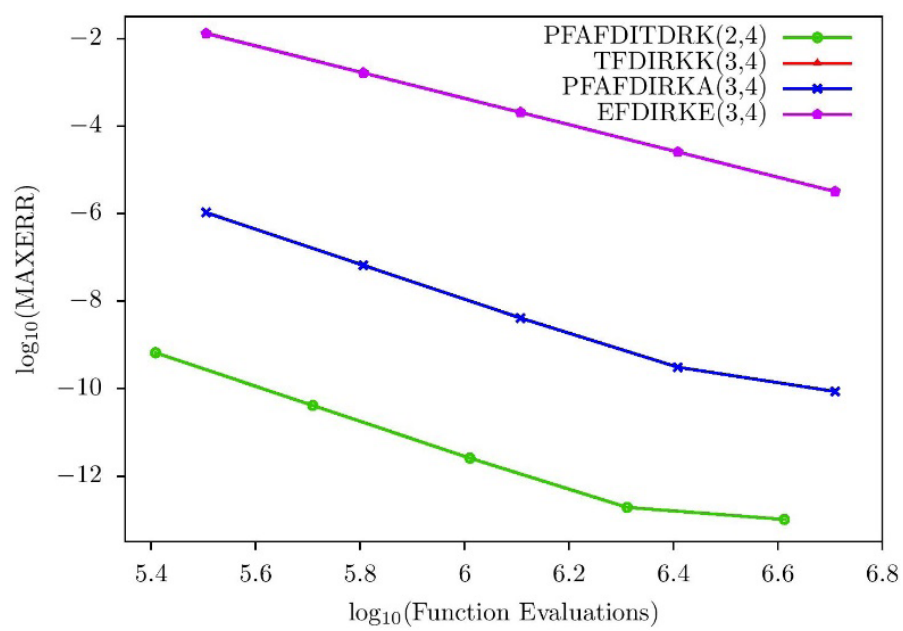

FIGURE 13. The curve for the almost periodic problem (Problem 3) with time step $\Delta \mathrm{t}=1.0 / 2^{i}, i=5, \ldots, 9$

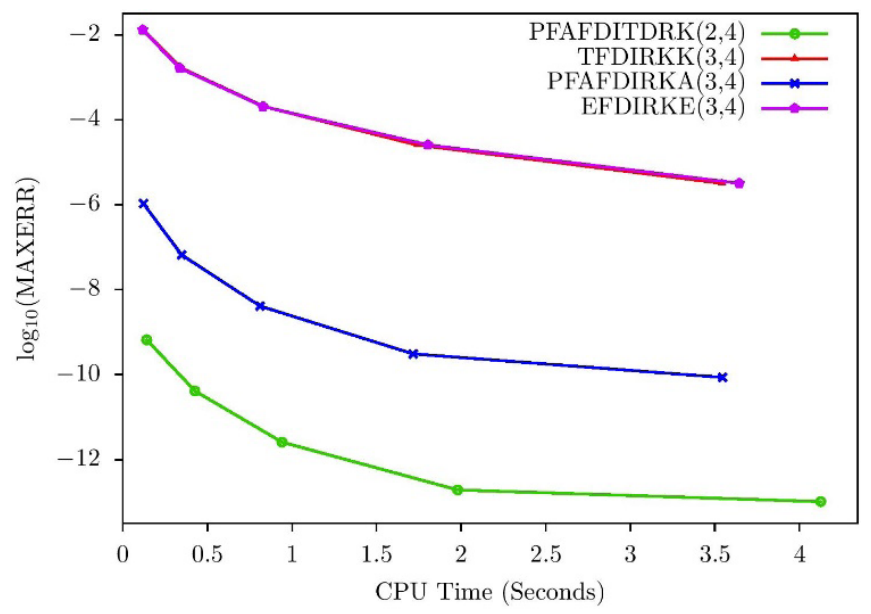

FIGURE 14. The curve for the almost periodic problem (Problem 3 ) with time step $\Delta \mathrm{t}=1.0 / 2^{i}, i=5, \ldots, 9$

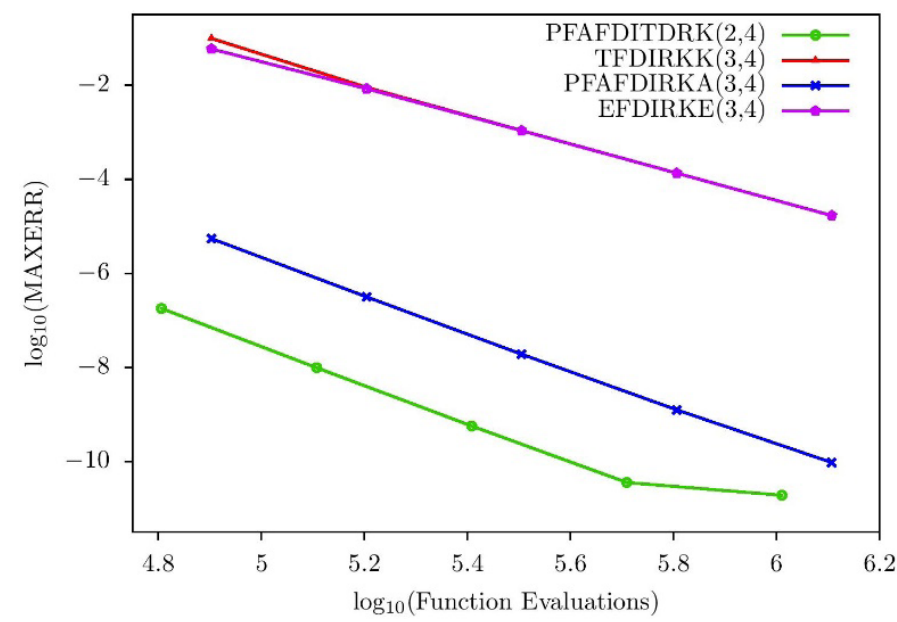

FIGURE 15. The curve for the Duffing problem (Problem 4) with time step $\Delta \mathrm{t}=1.0 / 2^{i}, i=3, \ldots, 7$ 


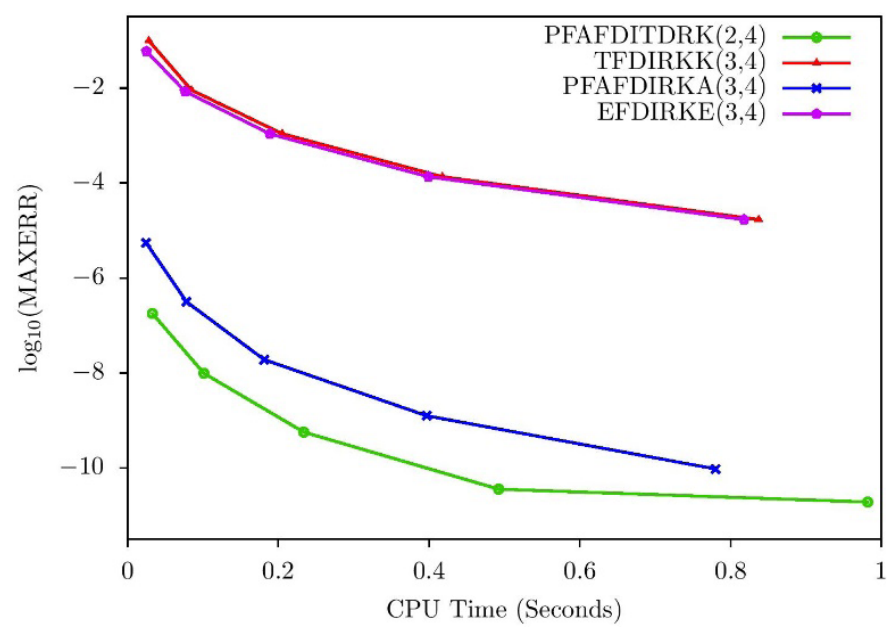

FIGURE 16. The curve for the Duffing problem (Problem 4) with time step $\Delta \mathrm{t}=1.0 / 2^{i}, i=3, \ldots, 7$

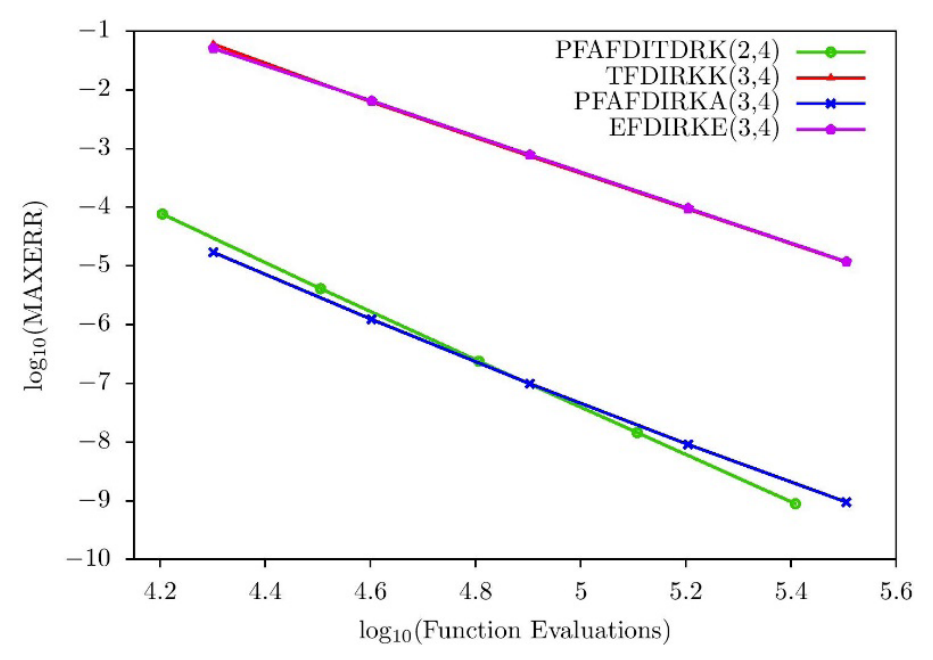

FIGURE 17. The curve for the Prothero-Robinson problem

(Problem 5) with time step $\Delta \mathrm{t}=1.0 / 2^{i}, i=1, \ldots, 5$

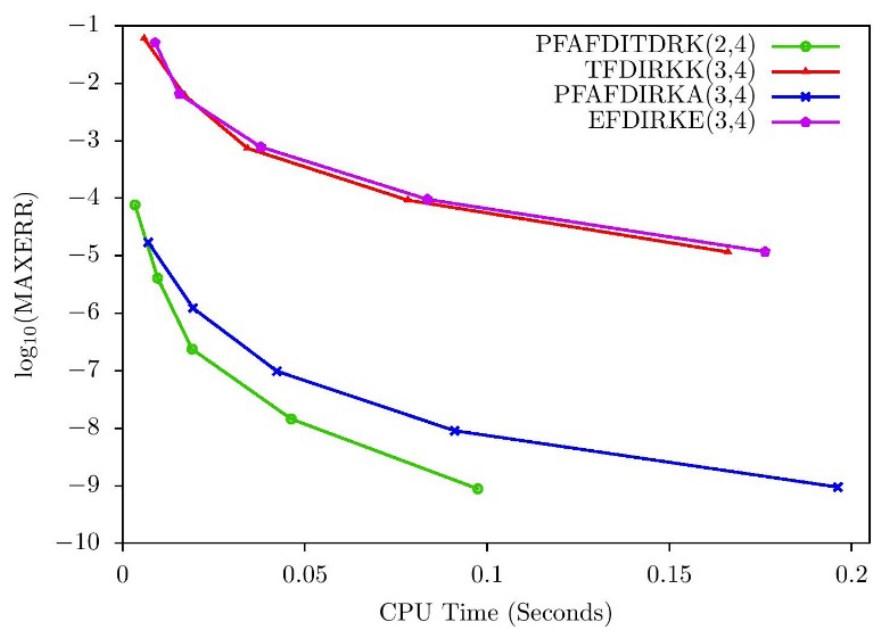

FIGURE 18. The curve for the Prothero-Robinson problem (Problem 5) with time step $\Delta \mathrm{t}=1.0 / 2^{i}, i=1, \ldots, 5$ 


\section{DISCUSSION}

The numerical results has shown the standard properties of the proposed phase-fitted and amplification-fitted DITDRK method, PFAFDITDRK $(2,4)$ which was obtained earlier. Several well-known existing RK methods are chosen as the comparison with the proposed method. The energy error at every integration point can be seen in Figure 3. Conservation of energy is succeeded by the phasefitted and amplification-fitted DITDRK method when it experienced smaller amount of energy error compared to TFDIRKK $(3,4)$, PFAFDIRKA $(3,4)$ and EFDIRKE $(3,4)$. The $\log$ number of global error against the time of integration for different time step, are plotted for distinct problems as shown in Figures 4-8. From Figures 4-7, it is identified that global error developed by the PFAFDITDRK $(2,4)$ method is smaller compared to $\operatorname{TFDIRKK}(3,4), \operatorname{PFAFDIRKA}(3,4)$ and EFDIRKE $(3,4)$. Meanwhile in Figure 8, the global error between PFAFDITDRK $(2,4)$ and PFAFDIRKA $(3,4)$ are rather close between one another but still the proposed method has the smallest global error.

Next, a long period of integration of the global error and the efficiency of the method are plotted. The log of the maximum global error versus the logarithm number of function evaluations and CPU time is plotted as given in Figures 9-18 to show the accuracy of the designed method. From Figures 9-18, the global error produced by PFAFDITDRK $(2,4)$ method is smaller compared to the same order existing RK methods. In Figures 12, 14 and 16 , PFAFDITDRK $(2,4)$ takes longer CPU time compared to other existing RK methods due to its method complexity which is caused by the existence of the extra $g$ to be evaluated at every step. In Figure 17, at the beginning of the graph, PFAFDITDRK $(2,4)$ has slightly bigger maximum global error compared to PFAFDIRKA $(3,4)$. As the value of $\Delta \mathrm{t}$ decreases, PFAFDITDRK $(2,4)$ has smaller maximum global error compared to PFAFDIRKA(3,4). From Figures 9-18, it can be seen that PFAFDITDRK $(2,4)$ method has the smallest maximum global error and the least amount of function evaluations per step.

One of the disadvantages of the derived method is that it is not suitable for solving stiff oscillatory or highly oscillatory problems which required the need of P-stable or strongly stable method. Therefore, we suggested that in the future work, the derivation of P-stable PFAFDITDRK is considered when one tries to solve stiff oscillatory or highly oscillatory problems.

Based on the phase-fitted and amplification-fitted property, the fitted property works well in solving linear problems but is not suitable in solving non-linear problems.
Hence, we did not include non-linear problem in the problems tested.

\section{CONCLUSION}

In this area of study, a fourth-order phase-fitted and amplification-fitted DITDRK method of has been proposed. Based on the numerical experiments, we can simplified that the proposed PFAFDITDRK $(2,4)$ method is more promising than any of the other well-known existing DIRK methods with trigonometrically-fitted and phasefitted and amplification-fitted property in terms of efficiency and accuracy as well as the number of function evaluations per step.

\section{ACKNOWLEDGEMENTS}

We are grateful to the Institute of Mathematical Research (INSPEM) and the Department of Mathematics and Statistics, Universiti Putra Malaysia for their continuous support and guidance throughout the research work. This study was supported financially by the Fundamental Research Grant Scheme (Ref. No. FRGS/1/2018/STG06/ UPM/02/2) awarded by the Malaysia Ministry of Education and MyBrainSc.

\section{REFERENCES}

Adel, F., Senu, N., Ismail, F. \& Majid, Z.A. 2016. New efficient phase-fitted and amplification-fitted Runge-Kutta method for oscillatory problems. International Journal of Pure and Applied Mathematics 107(1): 69-86.

Ahmad, N.A., Senu, N. \& Ismail, F. 2016. Phase-fitted and amplification-fitted diagonally implicit Runge-Kutta method for the numerical solution of periodic problems. Asian Journal of Mathematics and Computer Research 12(4): 252-264.

Anastassi, Z.A. \& Simos, T.E. 2005. Trigonometrically-fitted Runge-Kutta methods for the numerical solution of the Schrödinger equation. Journal of Mathematical Chemistry 37(3): 281-293.

Atkinson, K., Han, W. \& Stewart, D.E. 2009. Numerical Solution of Ordinary Differential Equations. Haboken, New Jersey: John Wiley \& Sons, Inc. pp. 67-94.

Chan, R.P. \& Tsai, A.Y. 2010. On explicit two-derivative RungeKutta methods. Numerical Algorithm 53: 171-194.

Chen, Z., Li, J., Zhang, R. \& You, X. 2015. Exponentially fitted two-derivative Runge-Kutta methods for simulation of oscillatory genetic regulatory systems. Computational and Mathematical Methods in Medicine 2015: 689137.

Chen, Z., You, X., Shu, X. \& Zhang, M. 2012. A new family of phase-fitted and amplification-fitted Runge-Kutta type methods for oscillators. Journal of Applied Mathematics 2012: Article ID. 236281. 
Demba, M.A., Senu, N. \& Ismail, F. 2016a. Trigonometricallyfitted explicit four-stage fourth-order Runge-Kutta-Nyström method for the solution of initial value problems with oscillatory behavior. Global Journal of Pure and Applied Mathematics 12(1): 67-80.

Demba, M.A., Senu, N. \& Ismail, F. 2016b. Fifth-order fourstage explicit trigonometrically-fitted Runge-Kutta-Nyström methods. In Recent Advances in Mathematical Sciences, edited by Kılıçman, A., Srivastava H.M., Mursaleen, M. \& Majid, Z.A. Singapore: SpringerNature. pp. 27-36.

Ehigie, J.O., Diao, D., Zhang, R., Fang, Y., Hou, X. \& You, X. 2018. Exponentially-Fitted symmetric and symplectic DIRK methods for oscillatory hamiltonian systems. Journal of Mathematical Chemistry 56(4): 1130-1152.

Fang, Y., You, X. \& Ming, Q. 2013. Exponentially fitted twoderivative Runge-Kutta Methods for the Schrödinger equation. International Journal of Modern Physics C 24(10): 1350073.

Fawzi, F.A., Senu, N., Ismail, F. \& Majid, Z.A. 2015. A phasefitted and amplification-fitted modified Runge-Kutta method of fourth order for periodic initial value problems. research and education. In 2015 International Conference on Research and Education in Mathematics (ICREM7). IEEE. pp. 25-28.

Henrici, P. 1962. Discrete Variable Methods in Ordinary Differential Equations. New York: John Wiley \& Sons, Inc.

Kalogiratou, Z. 2013. Diagonally implicit trigonometrically-fitted symplectic Runge-Kutta methods. Applied Mathematics and Computation 219(14): 7406-7412.

Konguetsof, A. \& Simos, T.E. 2003. An exponentially-fitted and trigonometrically-fitted method for the numerical solution of periodic initial-value problems. Computers \& Mathematics with Applications 45(1-3): 547-554.

Kosti, A.A., Anastassi, Z.A. \& Simos, T.E. 2012a. An optimized explicit Runge-Kutta-Nyström method for the numerical solution of orbital and related periodical initial value problems. Computer Physics Communications 183(3): 470-479.

Kosti, A.A., Anastassi, Z.A. \& Simos, T.E. 2012b. Construction of an explicit Runge-Kutta-Nyström method with constant coefficients and of a phase-fitted and amplification-fitted explicit Runge-Kutta-Nyström method for the numerical solution of the Schrödinger Equation. In AIP Conference Proceedings. AIP Publishing LLC. 1504(1): 1185-1187.

Lambert, J.D. 1991. Numerical Methods for Ordinary Differential Systems: The Initial Value Problem. Baffins Lane, Chichester: John Wiley \& Sons Ltd. pp. 149-212.

Pokorny, P. 2009. Continuation of periodic solutions of dissipative and conservative systems: Application to elastic pendulum. Mathematical Problems in Engineering 2009: Article ID. 104547.
Stiefel, E. \& Bettis, D.G. 1969. Stabilization of Cowell's method. Numerische Mathematik 13(2): 154-175.

Suli, E. \& Mayers, D.F. 2003. An Introduction to Numerical Analysis. Trumpington Street, Cambridge: Cambridge University Press.

Simos, T.E. 1998. An exponentially-fitted Runge-Kutta method for the numerical integration of initial-value problems with periodic or oscillating solutions. Computer Physics Communications 115(1): 1-8.

Van de Vyver, H. 2007. An explicit Numerov-type method for second-order differential equations with oscillating solutions. Computers \& Mathematics with Applications 53(9): 13391348 .

van der Houwen, P.J. \& Sommeijer, B.P. 1987. Explicit RungeKutta (-Nyström) methods with reduced phase errors for computing oscillating solution. SIAM Journal on Numerical Analysis 24(3): 595-617.

Zhang, Y., Che, H., Fang, Y. \& You, X. 2013. A new trigonometrically fitted two-derivative Runge-Kutta method for the numerical solution of the Schrödinger equation and related problems. Journal of Applied Mathematics 2013: Article ID. 937858.

Norazak Senu, Nur Amirah Ahmad*, Zarina Bibi Ibrahim \& Mohamed Othman

Institute for Mathematical Research

Universiti Putra Malaysia

43400 UPM Serdang, Selangor Darul Ehsan

Malaysia

Norazak Senu \& Zarina Bibi Ibrahim

Department of Mathematics and Statistics

Faculty of Science

Universiti Putra Malaysia

43400 UPM Serdang, Selangor Darul Ehsan

Malaysia

Mohamed Othman

Department of Communication Technology and Network

Faculty of Computer Science and Information Technology

Universiti Putra Malaysia

43400 UPM Serdang, Selangor Darul Ehsan

Malaysia

*Corresponding author; email: nuramirah_ahmad@yahoo.com

Received: 26 June 2020

Accepted: 27 October 2020 